\title{
DIALOG DER KIRCHE MIT DER WELT IN DER NACHPATRISTISCHEN ZEIT: DIE THEOLOGIE DES BISCHOFSAMTES AUF KONZILIEN VON TRIENT, I. UND II. VATIKANUM. SKIZZE EINER (MÖGLICHEN) LEKTÜRE AUS ORTHODOXER SICHT
}

Lucian D. Colda*

Abstract: The dialogue of the Church with the world in the post-patristic period: the theology of the episcopal ministry at the Council of Trent, the First Vatican Council and the Second Vatican Council. An outline of a (possible) lecture from the Orthodox perspective. One of the most significant aspects of the ecumenical dialogue regards the episcopal ministry. This dialogue also reveals that, unfortunately, over the centuries, the separation between the Christians of the various Churches and ecclesial communities was not static, but it permanently became more and more profound, especially concerning the understanding of the learning about the Church and, inherently, of the sacerdotal ministry. If between the Orthodox and the Catholic Churches there is an agreement in principal on the continuity with the Una Sancta Catholica et Apostolica Church of the first millennium, the situation is different when comes about the ecclesiological models offered by the theology of a Protestant tradition. Things become even more clear when they are looked at by the point of view offered by the learning about the episcopal ministry that the Catholic theology of the second Vatican Council perceives as being into a continuity with that of the Council of Trent and the first Vatican Council. This short study aims to emphasize an alternative reading of this theology from an Orthodox perspective, which is intented to be a fraternal and irenic one.

Keywords: the Orthodox Church, the Roman Catholic Church, episcopal ministry, the pope, the Council of Trent, the First Vatican Council, the Second Vatican Council, development, continuity, sacramentum ordinis.

\footnotetext{
* Ph.D., Assistant Professor, Faculty of Orthodox Theology, at "1 Decembrie 1918” University, Alba Iulia, Romania.
} 


\section{Zum Geleit: Die Theologie des Bischofsamtes im Kontext des ökumenischen Gesprächs ${ }^{1}$}

Eine der wichtigsten Fragen im heutigen ökumenischen Dialog, die in einem sehr engen Zusammenhang mit der Ekklesiologie steht, betrifft das kirchliche Amt. Sehr oft hört man, die Amtsfrage sei wesentlich im ökumenischen Dialog, ja sogar sie gehöre zu den dornigen Fragen im ökumenischen Gespräch unserer Tage $^{2}$. Bei einer solchen Behauptung scheint es so, als wäre nur die amtliche Funktion allein entscheidend, während die konkrete Ausübung dieser Funktion durch eine Person sekundär ist. Am deutlichsten kommen diese Gedanken und Überlegungen in den heutigen ökumenischen Gesprächen zum Ausdruck und werden vor allem von der protestantischen Theologie vertreten, welche die Kritik und Infragestellung des bisherigen Verständnisses von Amt und Ordination nicht nur als notwendig, sondern auch als eine große Chance zur Überwindung des angeblichen Monopols eines

${ }^{1}$ Man berücksichtige dazu auch unsere Studien: Episkopé und Bischofsamt nach Gunther Wenz. Überlegungen aus orthodoxer Sicht bezüglich einer zeitgenössischen evangelisch-lutherischen Auffassung des Bischofsamtes, in: Anuarul Facultăţii de Teologie Ortodoxă Andrei Şaguna - Sibiu,VI (XXXI)/2005-2006 (Sibiu 2008) 73-96 und Aspekte der Theologie des kirchlichen Amtes im ökumenischen Kontext mit Berücksichtigung der Konzeption von Dumitru Stăniloae bezüglich des Bischofsamtes, in „Anuarul Facultăţii de Teologie Ortodoxă Andrei Şaguna - Sibiu", Nr. VIII (XXXIII)/2007-2008 (Sibiu 2009) 96-118.

Die Abkürzungen dieser Studie richten sich nach: (a) Theologische Realenzyklopädie. Abkürzungsverzeichnis, zusammengestellt von S. M. SCHWERTNER (Berlin/New York 21994) und (b) Lexikon für Theologie und Kirche. Abkürzungsverzeichnis, begründet von M. BUCHBERGER und herausgegeben von W. KASPER zusammen mit K. BAUMGARTNER, H. Bürkle, K. Ganzer, K. Kertelge, W. KorfF, P. Walter (Freiburg i. Br./Basel/Rom/Wien 31993).

${ }^{2}$ G. GorscheneK, Vorwort, in: W. SANDERS (Hrsg.), Bischofsamt - Amt der Einheit: ein Beitrag zum ökumenischen Gespräch (München 1983) 7. 
akademisch vorgebildeten Einzelnen als Träger des Amtes ${ }^{3}$ sieht. Des Weiteren sollten die Kirchen eigentlich nicht mehr an einer Engführung des oben erwähnten Monopols festhalten, sondern sich neu an der Vielfalt vor (sic!) ordinationsfähigen und ordinationsbedürftigen Diensten und Ämtern ${ }^{4}$ orientieren.

Man muss feststellen, dass die im Laufe der Zeit zwischen den verschiedenen Konfessionen, Kirchen und kirchlichen Gemeinschaften zahlreich entstandenen, aber misslungenen Einigungsversuche gezeigt haben, dass die immer tiefere Entfremdung zwischen den Kirchen des Ostens und des Westens ${ }^{5}-$ was sich ja allerdings auch in der Unterbrechung der eucharistischen Gemeinschaft und im Fortdauer dieser Situation zu erkennen liesst nicht zuletzt eine Frage der Ekklesiologie und des kirchlichen Amtes ist; und wenn man vom kirchlichen Amt spricht, wird man insbesondere auf das Bischofsamt verwiesen. Erst in der zweiten Hälfte des 20. Jahrhunderts, nach Jahrhunderten der Trennung, kam es zu substanziellen Änderungen der Verhältnisse zwischen den verschiedenen Kirchen der Christenheit, insbesondere zwischen der römisch-katholischen Kirche und der orthodoxen Kirche des

3 G. Hage, Vorwort, in: A. Völker/K. Lehmann/H. Dombois (Hrsg.), Ordination heute (= KZPH 5) (Kassel 1972) 7.

${ }^{4}$ Ebd. 7.

5 Auf diese Situation weist auch das „Dekret über den Ökumenismus 'Unitatis redintegratio" (= UR; S. dazu: LThK.E (1967) II: Das zweite Vatikanische Konzil. Konstitutionen, Dekrete und Erklärungen. Lateinisch und deutsch 40 (41)-122 (123); hier UR 14 und 19) hin, wenn es behauptet: „Die Kirchen des Orients und des Abendlandes sind Jahrhunderte hindurch je ihren besonderen Weg gegangen. (...) Da jedoch diese Kirchen und Kirchlichen Gemeinschaften wegen ihrer Verschiedenheit nach Ursprung, Lehre und geistlichem Leben nicht nur uns gegenüber, sondern auch untereinander nicht wenige Unterschiede aufweisen, so wäre es eine überaus schwierige Aufgabe, sie recht zu beschreiben, was wir hier zu unternehmen nicht beabsichtigen. (...) Dabei muß jedoch anerkannt werden, daß es zwischen diesen Kirchen und Gemeinschaften und der katholischen Kirche Unterschiede von großem Gewicht gibt, nicht nur in historischer, soziologischer, psychologischer und kultureller Beziehung, sondern vor allem in der Interpretation der offenbarten Wahrheit" (Hervorhebungen von mir). 
byzantinischen Ritus' ${ }^{6}$. Der zwischen den Orthodoxen und den Katholiken entstandene theologische Dialog gab den Gesprächspartnern Anlass, sich wieder als Brüder und Schwestern im Glauben zu betrachten und gleichzeitig zu entdecken, dass das, was sie verbindet, mehr ist als jenes, was sie trennt. Infolgedessen kann man heute feststellen, dass viele theologische Themen einer erneuten und gemeinsamen Erörterung bedürfen. Unter all diesen Themen befindet und erweist sich als eine der wichtigsten Fragen, ja sogar als Schlüssel zukünftiger ekklesiologischer Konvergenzen, die Theologie des Bischofsamtes. Vorweg muss man aber feststellen, dass eine richtige Theologie des Bischofsamtes eines richtigen Verständnisses des Begriffs des kirchlichen Amtes bedarf.

Die Frage nach dem kirchlichen Amt in der Kirche, genauer: wie sich das Amt an sich und die ausübende Person dieses Amtes bzw. der funktionale Charakter des Amtes und das personale Element dieses Amtes zueinander verhalten, oder was für eine Relevanz die apostolische Sukzession und der sakramentale Charakter des ordinierten Amtes hat, ist und bleibt eine Herausforderung gegenwärtiger ökumenischer Tagungen; sie wird berücksichtigt nicht nur unter ihren systematisch-dogmatischen Aspekten, sondern sie bezieht auch Elemente der biblischen Exegese und der Dogmen- bzw. Kirchengeschichte in diese Analyse mit ein. Auch das II. Vatikanische Konzil, das sich als Entwicklung und zugleich Kontinuität des Tridentinums, bzw. des I. Vatikanums versteht, hat sich in mehreren Dekreten, insbesondere im oben genannten Dekret über den Ökumenismus 'Unitatis redintegratio' zur christlichen Einheit geäussert und auf die Amtsfrage als eines der grössten Hindernisse auf dem Weg zur Einheit aller Christen hingewiesen. Aus dieser Sicht und mit Bezug auf die hierarchische Struktur der Kirche unter dem dreifachen Amt des

${ }^{6}$ Vgl. T. BREMER/J. Oeldemann/D. StOltManN (Hrsg.), Orthodoxie im Dialog. Bilaterale Dialoge der orthodoxen und der orientalisch-orthodoxen Kirchen 1945-1997. Eine Dokumentensammlung (= SophQÖTh 32) (Trier 1999) 24. 
Apostelkollegiums mit und unter Petrus drückt sich das Dekret über den Ökumenismus 'Unitatis redintegratio' folgendermassen aus:

„Um nun diese seine heilige Kirche überall auf Erden bis zum Ende der Zeiten fest zu begründen, hat Christus das Amt der Lehre, der Leitung und der Heiligung dem Kollegium der Zwölf anvertraut. Unter ihnen hat er den Petrus ausgewählt, auf dem er nach dem Bekenntnis des Glaubens seine Kirche zu bauen beschlossen hat. (...) Dies ist das heilige Geheimnis der Einheit der Kirche in Christus und durch Christus, indes der Heilige Geist die Mannigfaltigkeit der Gaben schafft. (...) In dieser einen und einzigen Kirche Gottes sind schon von den ersten Zeiten an Spaltungen entstanden, die der Apostel aufs schwerste tadelt und verurteilt; in den späteren Jahrhunderten aber sind ausgedehntere Verfeindungen entstanden, und es kam zur Trennung recht großer Gemeinschaften von der vollen Gemeinschaft der katholischen Kirche, oft nicht ohne Schuld der Menschen auf beiden Seiten. (...) Da es zwischen ihnen und der katholischen Kirche sowohl in der Lehre und bisweilen auch in der Disziplin wie auch bezüglich der Struktur der Kirche Diskrepanzen verschiedener Art gibt, so stehen sicherlich nicht wenige Hindernisse der vollen kirchlichen Gemeinschaft entgegen, bisweilen recht schwerwiegende, um deren Überwindung die ökumenische Bewegung bemüht ist"?

Trifft das heute aber immer noch zu? Oder sind inzwischen schon alle Grenzen überwunden worden? Das Konzil hat weder ein letztes, endgültiges Wort noch ein absolutes Entweder-Oder ausgesprochen. Wenn man akzeptiert, dass das Amt zum Wesen der Kirche gehört, und dass es sich bei der Ordination zum Amt um ein sakramentales Geschehen und nicht um eine blosse Einführung in irgendeine Funktion handelt, dann bedarf es einer sorgfältigen Darstellung der jeweiligen Standpunkte heute und des Versuchs einer kritischen Stellungnahme dazu .

\footnotetext{
${ }^{7}$ UR 2-3 (Hervorhebungen von mir).

${ }^{8}$ H. SCHÜTTE, Amt, Ordination und Sukzession im Verständnis evangelischer und katholischer Exegeten und Dogmatiker der Gegenwart sowie in Dokumenten ökumenischer Gespräche (Düsseldorf 1974) 10.
} 
Aufgrund der verschiedenen Darstellungen über das geistliche Amt in den drei grossen Konfessionen ist es schon ersichtlich, wie viele Unterschiede in den Gesamtkonzepten dieser drei Konfessionen vorkommen. Gerade deswegen sind in den letzten Jahren über das kirchliche Amt verschiedene Gespräche geführt worden mit dem Ziel, all diese Unterschiede zu erörtern und zu fragen, wie man zu einer Einigung und einer gegenseitigen Anerkennung gelangen kann. Leider kann man aus den vielen entstandenen Dokumenten und Konsenstexten keine derartige Einigung feststellen, vielmehr sind die festgestellten Gemeinsamkeiten nur als Auslegungstendenzen zu verstehen. Fragen wie apostolische Sukzession, Interpretation des Apostolischen und Ordination als Weihesakrament, die mit der Amtsproblematik tief verbunden sind, bedürfen immer noch einer grundsätzlichen Erörterung. Man merkt dabei aber auch, dass sich katholischerseits seit dem II. Vatikanischen Konzil durchaus eine Entwicklung im Verständnis des kirchlichen Amtes zeigt ${ }^{9}$, die weitere Annährungen, insbesondere mit der orthodoxen Theologie innerhalb des auf verschiedene Ebenen geführten theologischen Dialogs, als möglich erscheinen lässt. Infolgedessen ist es leicht zu verstehen, wie dringend und notwendig eine Fortsetzung des im 20. Jahrhundert entstandenen orthodox-katholischen Dialogs und eine gemeinsame Theologie des Bischofsamtes aufgrund eines richtigen Verständnisses des kirchlichen Amtes ist. Auf eine solche Forderung weisen nicht zuletzt auch die in den letzten 50 Jahren veröffentlichen lehramtlichen Dokumente der katholischen Kirche und verschiedene andere Artikel, Aufsätze, Kommentare zu den Konzilsdokumenten, Sammelbände und Werke katholischer, orthodoxer und protestantischer Provenienz hin ${ }^{10}$.

${ }^{9}$ Vgl. C. H. RATSCHOW, Amt/Ämter/Amtsverständnis VIII, in: TRE 2 (1978) 618-620.

${ }^{10}$ S. dazu z.B.: S. ACKERMANN, Kirche als Person: zur ekklesiologischen Relevanz des personal-symbolischen Verständnisses der Kirche (= SSSTh 31) (Würzburg 2001); G. BAUSENHART, Das Amt in der Kirche: Eine notwendende Neubestimmung (Freiburg i. Br./Basel/Wien 1999); M. BRUN, Orthodoxe Stimmen zum II. Vatikanum unter besonderer Berücksichtigung der Kirchenkonstitution, Kp. I-III. Ein Beitrag zur Überwindung der 


\section{Entwicklung und Kontinuität: Tridentinum, I. und II. Vatikanum - die Notwendigkeit einer theologischen Zusammenschau}

Eine der wichtigsten theologischen Aussage entspricht der Überzeugung, dass das Bischofsamt sowohl in der katholischen als auch in der orthodoxen Kirche in einer ununterbrochenen Kontinuität seit dem 2. Jahrhundert bezeugt ist. Diese Kontinuität hat aber durch die Zeit hindurch auch eine legitime Entwicklung mit sich gebracht. Bereits vor der Wende zum 3. Jahrhundert hat sich das Bischofsamt in der ganzen Kirche des Ostens und des Westens durchgesetzt. Sogar nach der schmerzlichen kirchlichen Trennung von Ost und West wurde es im Osten und im Westen gegenseitig anerkannt, denn sowohl die Kirche des byzantinischen Ritus als auch die Kirche des lateinischen bzw. römischen Ritus hatten in ihm ein wesentliches, ja sogar das wesentliche Element der Ekklesiologie schlechthin gesehen. Die ersten Kontroversen darüber sind erst mit der Reformation entstanden, als sich die infolge dieses Ereignisses

Trennung (= ÖB 18) (Freiburg i. Ü. 1987); Y. CONGAR (Hrsg.), Das Bischofsamt und die Weltkirche (Stuttgart 1964); R. FRIELING, Amt: Laie Pfarrer - Priester - Bischof - Papst (= BenshH 99; OeS 13) (Göttingen 2002); A. GANOCZY, Amt und Apostolizität. Zur Theologie des kirchlichen Amtes bei Calvin auf dem Hintergrund der gegenwärtigen ökumenischen Diskussion (= VIEGM 59) (Wiesbaden 1975); C. H. RATSCHOW, Amt/Ämter/Amtsverständnis VIII, in: TRE 2 (1978); W. SANDERS (Hrsg.), Bischofsamt - Amt der Einheit: ein Beitrag zum ökumenischen Gespräch (München 1983); H. SCHÜTTE, Amt, Ordination und Sukzession im Verständnis evangelischer und katholischer Exegeten und Dogmatiker der Gegenwart sowie in Dokumenten ökumenischer Gespräche (Düsseldorf 1974); W. StÄHLIN/J. H. LERCHE/E. FinCKE/L. Klein/K. RAHNER (Hrsg.), Das Amt der Einheit. Grundlegendes zur Teologie des Bischofsamtes (Stuttgart 1964) 77-190; K. KocH, 25 Jahre nach dem Zweiten Vatikanischen Konzil: welche Kirche hat Zukunft? Kontroverse Lektüre des Zweiten Vatikanischen Konzils und katastrophaler Streit der Kirchenbilder und wie die gegenwärtige Kirchenkrise überwunden müsste. Ein programmatischer Beitrag, in: SKZ 1 (1999) 2-13. 
entstandenen kirchlichen Gemeinschaften aus der bischöflichen altkirchlichen Ordnung herauslösten. Das Reform-Konzil von Trient hat zwar diese bischöfliche Ordnung erneut bestätigt, aber wegen der inzwischen entstandenen innerkatholischen Schwierigkeiten kam es nicht mehr $\mathrm{zu}$ einer endgültigen und zugleich befriedigenden Theologie des Bischofsamtes ${ }^{11}$, und deswegen blieb der Bischof in seiner theologischen Stellung umstritten ${ }^{12}$.

Einige wichtige Aspekte der Theologie des Bischofsamtes, wie z.B. seine kanonistisch-jurisdiktionelle Dimension im Bezug zum Papstamt und seine sich daraus ergebende ekklesiale Einordnung, blieben nicht zureichend geklärt. Das Konzil hat ja schliesslich das Grundverständnis des sacramentum ordinis bewusst nicht entschieden, und deswegen konnte und wollte es auch kein endgültiges dogmatisch verbindliches Verständnis des Bischofsamtes festlegen. Es hat sich nur auf den notwenigen Rahmen zur Verurteilung reformatorischer Irrtümer bezüglich der OrdoTheologie beschränkt. Deswegen konnte sich auf dem Konzil nicht ein endgültiges Basisverständnis des Bischofsamtes durchsetzen. Dennoch zeigen die Debatten, die Reformdekrete und die erlassenen Kanones zweifelsohne die Tendenz der Konzilsväter, sich an einem bischöflich grundgelegten ordo $\mathrm{zu}$ orientieren. Die Dichotomie zwischen Theologie und Kanonistik bezüglich des Bischofsamtes wird in Trient zwar in dem Sinne überwunden, dass dem Bischof, unter besonderer Berücksichtigung einiger Konzepte und Ideen - wie z.B. die des Guten Hirten oder der persönlichen Residenz und der persönlichen und nicht einfach rein funktionalen Verpflichtung zur Seelsorge - positiv-kanonistische Rechte zugestanden wurden. Mit der Idee des Guten Hirten wollte das Konzil die potestas ordinis und die potestas iurisdictionis auf ihre ursprüngliche ekklesiologische

${ }^{11}$ Vgl. W. BREUNING, Das Verständnis des katholischen Bischofsamtes nach dem Zweiten Vatikanischem Konzil, in: W. SANDERS (Hrsg.), Bischofsamt Amt der Einheit. Ein Beitrag zum ökumenischem Gespräch (München 1983) 10.

12 Vgl. J. Freitag, Sacramentum ordinis auf dem Konzil von Trient. Ausgeblendeter Dissens und erreichter Konsens (= IThS 32) (Innsbruck/Wien 1991) 25. 
Aufgabe beziehen und auf diese Art und Weise dann beide über das gemeinsame Ziel einander zuordnen. So konnte auch der Dienst der Verkündigung seine Einordnung in den ordo finden, und auf diese Weise wurde also ein Wechsel von der Vollmachtsperspektive zu einem am Dienst orientierten Verständnis des sacerdotium, ein Wechsel zu einem für die Auferbauung der Kirche sehr nötigen und wichtigen, pastoral orientierten Ansatz des Bischofsamtes ermöglicht ${ }^{13}$.

Für die Konzilsväter war schon von Anfang an, ja sogar zur Zeit der Konzilseinberufung bereits klar, dass die ganze bischöfliche Autorität, durch welche sie den Dienst an der Kirche ausüben und durch welche sie für den Glauben Zeugnis ablegen, eigentlich in ihrer bischöflichen Weihe gründet und wurzelt, mithin an ihre eigene Person gebunden ist. Deswegen lässt sich die Ausübung dieser Autorität überhaupt nicht durch eine andere Person vertreten ${ }^{14}$, denn nicht dem Amt sondern der Person, die das Amt ausübt, erweist man die Ehre, denn sie hat den Vorrang. So lässt sich leichter erklären, warum gerade diese Konzeption auf der ersten Tagungsperiode des Konzils Anlass zu vielen Auseinandersetzungen bot. Für das heutige Verständnis erscheint als normal, dass der Bischof selbst, durch seinen persönlichen Einsatz seine Autorität und seinen apostolischen Auftrag innerhalb seiner Diözese ausübt bzw. erfüllt. In der Kirche des Mittelalters war es aber nicht selbstverständlich, und oft wurde die an den Bischof gebundene Amtspflicht als ablösbar von seiner Person betrachtet. Oft waren nicht die Diözesanbischöfe sondern ihre Generalvikare oder - falls es um die Erteilung der Weihen ging - die Titularbischöfe diejenigen, welche die dem Bischof reservierten Amtshandlungen übernahmen, während die Bischöfe selbst außerhalb ihrer Diözese weilten. Gerade aus diesen Gründen wurde

\footnotetext{
${ }^{13}$ Vgl. ebd. 386f.

${ }^{14}$ Vgl. H. JeDIN, Geschichte des Konzils von Trient 1: Der Kampf um das Konzil (Freiburg i. Br. 1949) 410.
} 
die auf der ersten Tagungsperiode diskutierte bischöfliche Residenzpflicht zum Angelpunkt der Kirchenreform ${ }^{15}$.

Mit diesen Gedanken hat das Konzil von Trient festgelegt, dass das einzige Kriterium für das richtige Verständnis des Bischofsamtes nur von der Hirtenaufgabe her abgeleitet werden kann. Die Hirtenaufgabe wird aber erst ins Licht gestellt mit und in der Weihe, oder anders gesagt: sie gründet in der Weihe, die den Kandidaten zum Bischof macht. Nicht die einfache Ernennung durch den Papst, die im Grunde genommen nur einen rein funktionalen Charakter hat, macht jemanden zum Bischof, sondern allein die Weihe. Dieses Kriterium benützten dann die Konzilsväter zur Herleitung der Übergeordnetheit der Bischöfe iure divino, denn sie, d.h. die Bischöfe, haben das aus der Natur des in der Weihe übertragenen Hirtenamtes. Das vollständige officium pastorale ist dem Bischof erst durch die Weihe übertragen, in der er die zwei Vollmachten, d.h potestas ordinis und potestas iurisdictionis, in vollkommener Weise empfängt, denn sonst kann er seiner Aufgabe nicht gerecht werden. Diese potestas pastoralis ist aber keineswegs eine potestas mundana, sondern sie wurzelt in Christus selbst, sie ist potestas Christi. Diese christologische, an die Person Jesu Christi gebundene Bestimmung des bischöflichen Hirtenamtes, ergibt sich aus seiner eigenen Aufgabe, dem Sorgen für das Heil der ihm anvertrauten Seelen. Genau so wie die Apostel von Christus selbst eingesetzt wurden, so werden auch die Bischöfe bis ans Ende der Zeit durch den in seiner Kirche immer gegenwärtigen Christus eingesetzt, der ihnen alles, was sie zur Erfüllung ihres Auftrages notwendig haben, gibt. Deswegen gehört all das zum Sein und Wesen des Bischofs und ist eng an seine eigene Person und an die Person Jesu Christi gebunden ${ }^{16}$, denn Christus bedient sich als von Gott bestellter neuer Hohepriester des Neuen Bundes der Priester dieses Neuen Bundes, in

${ }^{15}$ Zur Entfaltung dieser Problematik und den dazu auf dem Trienter Konzil entstandenen Diskussionen und Debatten s. ebenso: H. JEDIN, Geschichte des Konzils von Trient 2: Die erste Trienter Tagungsperiode 1545/47 (Freiburg i. Br. 1949) 269-315

${ }^{16}$ Vgl. J. FREITAG, Sacramentum ordinis 281-283. 
dem Sinne, dass er durch sie und in ihrem und in ihren Tun wieder auf eine sakramentale Weise gegenwärtig wird ${ }^{17}$. Man kann also sehen, dass trotz verschiedener Meinungen, die nicht selten heftige Diskussionen hervorgerufen haben, unter den Konzilsvätern eine grundlegende Meinung herrschte, die auf den personalen Dienstcharakter des kirchlichen Amtes, insbesondere des Bischofsamtes, hinweist ${ }^{18}$.

Aufgrund dieser Überlegungen wurde den Konzilsvätern klar, dass dem Bischof innerhalb der Kirche eine wichtige, ja sogar die zentrale Stelle zukommt, denn an ihm allein entscheidet sich das ganze Ordo-Verständnis, denn nur in ihm ist das Grundverständnis der Leitungsgewalt und des Ordo-Sakramentes zu gewinnen ${ }^{19}$. Grundsätzlich wurde über das Bischofsamt aber erst in der dritten Tagungsperiode entschieden, nachdem sich das Ringen darum in verschiedenen Schritten und Richtungen zunehmend entwickelt hat. Insbesondere hat die Frage der bischöflichen Rechte und ihrer Begründung das Konzil bis zum Ende in Spannung gehalten. So tauchten in der letzten Konzilsperiode einige heisse Fragen auf, wie z.B. ob der Bischof dem Priester ordinationsmässig übergeordnet sei, oder die Frage nach dem Verhältnis von potestas ordinis und potestas iurisdictionis, oder das Verhältnis von bischöflicher und päpstlicher Vollmacht ${ }^{20}$.

Wenn man anlässlich der Diskussion über das Weihesakrament die Lehre über das Bischofsamt nicht umgehen konnte, weil schon

${ }^{17}$ Vgl. K. J. BECKER, Der priesterliche Dienst 2: Wesen und Vollmachten des Priestertums nach dem Lehramt (= QD 47) (Freiburg i. Br./Basel/Wien 1970) 104.

${ }^{18}$ S. die Debatten während der zweiten Trienter Tagungsperiode bezüglich des Weihesakramentes. Vgl. dazu: H. JEDIN, Geschichte des Konzils von Trient 3: Bologneser Tagung (1547/48) - Zweite Trienter Tagungsperiode (1551/52) (Freiburg i. Br./Basel/Wien 1970) 350-358, insbesondere die Aussage des Augustiners Stephanus de Sestino (hier 351).

${ }^{19}$ Vgl. J. FreITAG, Schwierigkeiten und Erfahrungen mit dem ,sacramentum ordinis " auf dem Konzil von Trient, in: ZKTH 113 (1991) 51.

${ }^{20} \mathrm{Vgl}$. ebd. $41 \mathrm{f}$. 
zur Frage der Residenz bedeutende Schwierigkeiten hervortraten ${ }^{21}$, wurde auf der dritten Tagungsperiode die Frage des göttlichen Rechts des Bischofsamtes sorgfältig zur Diskussion gestellt, so versuchte man die Sakramentalität der Weihe aus der Offenbarung nachzuweisen ${ }^{22}$. Im Grunde genommen waren die Konzilsväter am Ende des Konzils nicht ganz zufrieden mit dem aus den Debatten entstandenen Bischofsspiegel ${ }^{23}$. Insgesamt lässt sich aber feststellen, dass die ganze Entwicklung der Ordo-Reform auf dem Konzil von Trient und insbesondere die Debatte über das Bischofsamt eine Ausrichtung auf die Seelesorge aufweist, selbst wenn sich die personale Dimension des Seelsorgeauftrags und die mit ihr verbundene personale Seelsorgevollmacht des Bischofs und des Bischofsamtes nicht ganz durchsetzen konnte, obwohl das schon die Zentralidee vieler Konzilsväter war. Wenn man zurück auf die erste Tagungsperiode des Konzils blickt, kann man merken, dass die Hauptbeschäftigung der Konzilsväter war, die Sakramentalität des ordo festzustellen und sie durch bestimmte Aussagen zu bekräftigen. Konkret und methodisch gründen diese Aussagen in den Zurückweisungen durch Martin Luther. Das Einheitsprinzip des ordo wurde dementsprechend in der Sakramentalität festgelegt, wenn auch nur formal. Die Einsetzung des ordo durch Jesus Christus selbst, die in der Weihe gegebene Geistmitteilung und die daraus entstandene Bezeichnung der Handlung als sacra ordinatio galten für die Konzilsväter als Kriterien dieser Sakramentalität. Deswegen gilt für das Konzil das Wesen des Priestertums als Befähigung zum Vollzug der sakramentalen Ordnung im Allgemeinen und nicht einfach als

21 Wegen der entstandenen heftigen Diskussionen musste sogar der Papst intervenieren, und infolgedessen wurde jegliche Diskussion über die Residenzpflicht der Bischöfe verboten. S. dazu: J. FREITAG, Schwierigkeiten und Erfahrungen 43.

${ }^{22}$ Vgl. H. JedIN, Geschichte des Konzils von Trient 4: Dritte Tagungsperiode und Abschlu $\beta / 4_{1}$ : Frankreich und der neue Anfang in Trient bis zum Tode der Legaten Gonzaga und Seripando (Freiburg i. Br./Basel/Wien 1975) $210 f$.

${ }^{23}$ S. dazu: ebd. $/ 4_{2}$ : Überwindung der Krise durch Morone, Schließung und Bestätigung (Freiburg i. Br./Basel/Wien 1975) 170f. 
Ansatz zum Verständnis des sacramentum ordinis im Sinne einer sazerdotalen Opferungsaufgabe ${ }^{24}$.

Die Auseinandersetzungen mit der Ordo-Theologie von Martin Luther ist in allen drei Tagungsperioden des Konzils geführt worden. Man kann die Haupteinwände Martin Luthers gegen das sacramentum ordinis überhaupt und gegen die Sakramentalität dieses ordo unter folgenden drei Punkten zusammenfassen ${ }^{25}$ :

a) Zwischen Priestern und Laien gibt es keinen Unterschied im Sinne eines Wesensunterschiedes und dazu auch keinen Standesunterschied, weil die christliche Brüderlichkeit wichtiger und grundlegender als der Akt der Ordination ist;

b) Die Priester können nicht für andere genugtun;

c) Der Verkündigungsauftrag der Kirche ist allein konstitutiv für das Wesen des sacramentum ordinis. Die Priester sind nur Diener des Wortes und nichts mehr; deswegen gibt es auch kein Weihesakrament.

Das Konzil hat diese Ansichten Martin Luthers bezüglich des sacramentum ordinis entschieden abgelehnt. Die Frage der Verkündigung war für die Konzilsväter auf der praktischkanonischen und nicht der dogmatischen Ebene angesiedelt. Deswegen konnte die Verkündigung nicht als theologisches Grundkriterium des ordo gelten. Was die lutherische Auffassung, die Priester können nicht für andere genugtun, anbelangt, haben die Konzilsväter diesen Einwand der Messopferfrage zugeordnet. Der geleugnete wesentliche Unterschied zwischen Laien und Priestern wurde vom Konzil als sakramentaler bestimmt und definiert. So erscheint dieser Unterschied in den Augen der Konzilsväter als Gottes- und nicht als Menschenwerk, also nicht als eine reine Funktion, die von der Kirche auf irgendeine Weise erfüllt wird. Zugleich kann man bei den Konzilsvätern auch eine andere Richtung feststellen, und zwar, dass die sazerdotale Perspektive von einer episkopalen abgelöst wurde. Nicht der Priester, sondern der Bischof

\footnotetext{
${ }^{24}$ Vgl. J. FreITAG, Sacramentum ordinis $359 f$.

${ }^{25}$ S. dazu auch: ebd. 360.
} 
wird zum Angelpunkt und zur Grundgestalt des ordo. So erscheint nun der Bischof als Spitze der Ordo-Hierarchie ${ }^{26}$. Die Konzilsväter bemühten sich zu zeigen, dass der Bischof grundsätzlich dem Priester - und das nicht nur aus einer rein kirchenrechtlichen Sicht, sondern auch auf sakramentaler Ebene - vorgeordnet ist. Eine lange und einseitige Betonung des Opfercharakters der Messe hatte bis zur Zeit des Konzils zur Folge die Konzentration des kirchlichen Amtes nur auf das Priesteramt, während das Bischofsamt im Hintergrund blieb. Das Konzil von Trient hat mit der episkopalen Perspektive die alte Tradition der Kirche endgültig wiederhergestellt ${ }^{27}$. Dieser Sicht entsprechend rückt der Bischof wieder zur Schlüsselgestalt des sacramentum ordinis auf, was logischerweise $\mathrm{zu}$ einer neuen Definierung der Stellung des Bischofs in der Kirche geführt hat. Was auf dem Konzil eigentlich entscheidend war, war der Anspruch der Väter auf die Christusunmittelbarkeit des Bischofsamtes. Dieser Anspruch der Konzilsväter konnte sich eigentlich problemlos im Verhältnis zu den Presbytern durchsetzten. Allein im Verhältnis zum Papst konnte er sich nicht durchsetzen, denn der Anspruch der römischen Bischofs, alleinige Jurisdiktionsquelle oder einziger Ort jurisdiktioneller Unmittelbarkeit zu Jesus Christus zu sein, wurde von den Konzilsväter nicht bestritten. So konnte man erstaunlicherweise im Trient von den Bischöfen sprechen, ohne den Papst zu nennen, wenn auch nur in einer sakramentalen Dimension. Die nachkonziliare durchgesetzte Kirchenreform konnte aber zu dem Ergebnis führen, in dem der Bischof - was eigentlich während des Konzils angedeutet wurde, wenn auch nicht erfolgreich - praktisch auch in Sache der Jurisdiktion in eine Schlüsselstellung aufgerückt worden ist, die ihnen am Anfang des Konzils nicht zugedacht war. Der entscheidende Punkt dafür war eigentlich das aus der

\footnotetext{
${ }^{26} \mathrm{Vgl}$. ebd. 360f.

${ }^{27}$ Vgl. M. Venard, Die katholische Kirche, in: M. VEnARd/(H. SMOLInSKY deutsche Ausgabe) (Hrsg.), Die Geschichte des Christentums 8: Die Zeit der Konfessionen (1530-1620/30) 269f.
} 
Hirtenaufgabe ergebende Postulat der Residenz der Bischöfe, das wie gezeigt - zum Angelpunkt der Kirchenreform wurde ${ }^{28}$.

Ebenso wie das Konzil von Trient wurde auch das Vatikanum I in einer Zeit der Irrtümer und Nöte einberufen, um sich mit ihnen zu befassen. Infolge verschiedener (nicht zuletzt politischer, das Verhältnis zwischen Staat und Kirche betreffender) Faktoren stellte sich schliesslich als Gegenstand des Konzils nur das Papsttum, seine Stellung und Autorität innerhalb der Kirche ${ }^{29}$, heraus. So erklärte das Konzil allein die Lehre über den Papst als verbindlich, obwohl ursprünglich die Entwürfe auch Aussagen über das Bischofsamt enthielten, die aber wegen des vorzeitigen Konzilsabbruchs nicht mehr zur Diskussion kamen. Auf jeden Fall war es nicht die Absicht der Konzilsväter, sich allein mit dem Papstamt zu beschäftigen ${ }^{30}$. Das Ziel des Konzils hätte eigentlich eine verbindliche Ekklesiologie mit wichtigen Aussagen über das Bischofsamt und dessen Verhältnis zum Papstamt sein sollen. Bedauerlicherweise ist es den Konzilsvätern nicht gelungen eine solche Ekklesiologie zu entwickeln. Bereits in Trient scheiterte eine solche Tendenz, obwohl zu erkennen war, dass der wichtigste Streitpunkt zwischen Katholiken und Protestanten die Lehre über die Kirche war. Das I. Vatikanische Konzil wollte eben diese Lehre aufgreifen und umfassend definieren. Leider kam von den 15 Kapiteln des vorgelegten Entwurfs über die Kirche nur das 9. Kapitel (über den Papst) zur Debatte. Die geplante Diskussion über die anderen Kapitel wurde durch die Vertagung des Konzils verhindert. Trotz dieser Vertagung kamen auch einige Aspekte über die Lehre des Bischofsamtes ans Licht. So lehrt das Konzil z.B., dass die Bischöfe Zeugen und Richter des Glaubens sind. Jeder Bischof bezeugt personal den Glauben seiner anvertrauten Herde und Kirche und zusammen mit ihr einen bestehenden consensus fidelium. Auch wenn die Unfehlbarkeit eines Konzils oder die des Papstes nicht von einer

\footnotetext{
${ }^{28}$ Vgl. J. FreitAG, Sacramentum ordinis 364-366.

${ }^{29}$ Vgl. C. BUtLeR/H. LANG (Übers.), Das I. Vatikanische Konzil (München ${ }^{2}$ 1961) 13 .

${ }^{30}$ Vgl. W. BREUnING, Das Verständnis des katholischen Bischofsamtes $10 \mathrm{f}$.
} 
Zustimmung der Gläubigen abhängig ist - denn sie haben nicht die Definitionen und Lehrentscheidungen $\mathrm{zu}$ billigen (consensus subsquens) - müssen sie sich trotzdem, ehe sie ihren Spruch fällen (consensus antecedens) vergewissern, dass sie der vorgegebenen Überzeugung der gesamten Kirche entsprechen. Deswegen sind die Bischöfe auf einem Konzil als iudices zu verstehen und als solche tätig. Aus diesem Grund ist eine Beteiligung der Laien am Konzil nicht zwingend nötig, denn die Bischöfe können aufgrund des ihnen zustehenden charisma veritatis und der göttlichen Einsetzung in solidum die Einheit im Glauben feststellen und die Irrtümer als solche erkennen und werfen ${ }^{31}$.

Wenn man nun die Lehre des I. Vatikanischen Konzils in ihrer Intention richtig zu verstehen versucht, stellt man fest, dass man die Problemstellung des Konzils nicht einfach auf zwei Termini - Papst bzw. Konzil - reduzieren darf, sondern man muss sie in einem grösseren ekklesiologischen Kontext betrachten. Natürlich ist es aber auch klar, dass das I. Vatikanum und seine Lehre vom Primat des Papstes einem schlecht verstandenem Konziliarismus, der die Oberhoheit eines Konzils über und gegen den Papst zum Rechtsprinzip machen wollte, widersprechen ${ }^{32}$. Zugleich muss man aber eindeutig sagen, dass die römischen Bischöfe der ersten Jahrhunderte keinen Primat im Sinne des I. Vatikanum ausgeübt haben. Erst seit dem 4. oder 5. Jahrhundert haben die römischen Päpste eine Petrustypologie für sich in Anspruch genommen. Auf jeden Fall kann man sagen, dass die Lehre des I. Vatikanischen Konzils über den Primat des römischen Papstes der Stellung der Bischofsamtes innerhalb der Kirche keinen Abbruch tun wollte, sondern sie gerade anerkennen, verteidigen und festigen wollte ${ }^{33}$.

\footnotetext{
${ }^{31}$ Vgl. C. BUTLER/H. LANG (Übers.), Das I. Vatikanische Konzil 527-529.

${ }^{32}$ Vgl. W. Breuning, Das Verständnis des katholischen Bischofsamtes 12.

33 S. dazu: Die Antworten auf die Circular-Depesche des Reichskanzlers Bismarck über die Auslegung der Konstitution „Pastor aeternus“ des I. Vatikanischen Konzils, Januar - März 1875:

a) Gemeinsame Erlärung der Bischöfe Deutschlands, Jan. - Febr. 1875 und
} 


\section{$15^{\text {th }}$ International Symposium on Science, Theology and Arts}

Genau so wie das Bischofsamt ist auch das Amt des römischen Bischofs zu verstehen, nämlich als Dienst an der Einheit, der notwendigerweise zugleich auch ein Dienst am Glauben ist ${ }^{34}$. Deswegen ist das Papsttum auf diese Einheit der Gesamtkirche und des Gesamtepiskopats wesenhaft verwiesen ${ }^{35}$, denn der Papst kann so wie das I. Vatikanische Konzil lehrt - nichts anderes sein als der Garant oder das Band des einen Glaubens und der Liebe, durch das alle Glieder der Kirche zusammengehalten werden ${ }^{36}$. Dennoch hatte das Konzil grosse Mühe, das Verhältnis zwischen der Vollmacht des Papstes, die als ordinaria, vera, immediata et episcopalis bezeichnet wurde, und der Vollmacht der Bischöfe zu bestimmen ${ }^{37}$. Am deutlichsten kann man das erkennen in den Texten der Constitutio prima de Ecclesia Christi ${ }^{38}$ und der wegen der geschichtlichen Umstände nicht mehr zur Diskussion gekommenen Constitutio secunda ${ }^{39}$, insbesonders in ihrem 4. Kapitel, das im Grunde

b) Apstolisches Schreiben „Mirabilis illa constantia“ an die Bischöfe Deutschlands, 4. März 1875, in: DH 3112-3117.

${ }^{34}$ Vgl. W. KASPER, Der Bischof von Rom als Diener der Einheit, in: W. SANDERS (Hrsg.), Bischofsamt - Amt der Einheit: ein Beitrag zum ökumenischen Gespräch (München 1983) 91.

${ }^{35}$ Vgl. H. U. von Balthasar, Der antirömische Affekt. Wie läßt sich das Papsttum in der Gesamtkirche integrieren (= HerBü 492) (Freiburg i. Br. 1974) 182.

${ }^{36}$ Vgl. dazu: Die dogmatische Konstitution „Pastor aeternus“ über die Kirche Christi, in: DH 3050-3052.

37 Vgl. G. BAUSENHART, Theologischer Kommentar zum Dekret über die Hirtenaufgabe der Bischöfe in der Kirche"Christus Dominus", in: HThKVatII, Bd. 3, 232f.

${ }^{38}$ DH 3050-3075.

${ }^{39}$ MANSI 53, 308A-317A. Der Titel dieser geplanten Konstitution, die zum Schluss 16 Canones enthielt, lautete eigentlich: "Schema constitutionis dogmaticae secundae de ecclesia Christi secundum reverendissimorum animadversiones reformatum". Die dazu gehörenden 10 Kapitel waren folgendermassen überschrieben:

I. De divina ecclesiae institutione;

II. Ecclesia a Christo institutam esse coetum fidelium;

III. Esse in ecclesia potestatem divinitus ordinatam;

IV. De ecclesiastica hierarchia; 
genommen wichtige Aussagen des Tridentinums wiederholt ${ }^{40}$. Die Intention des I. Vatikanischen Konzil war aber nicht, das ius divinum

\footnotetext{
V. De membris ecclesiae;

VI. Unam esse veram ecclesiam, neque extra eam salutem sperandam;

VII. De ecclesiastico magisterio;

VIII. De ecclesiastica jurisdictione;

IX. Ecclesiam esse verum regnum, divinum, immutabile et sempiternum;

X. Veram Christi ecclesiam non esse aliam nisi Romanam.
}

${ }^{40}$ MANSI 53, 310: „Sed neque omnes, qui in opus ministerii assumuntur, pari inter se potestate praediti sunt. Siquidem apostolica traditione patrumque consensu firmatum est, solis sacerdotibus prae ceteris ministris potestatam esse, corporis et sanguinis Domini sacramentum conficiendi atque fideles peccatorum vinculis solvendi; inter ipsos rursum sacerdotes episcopos, quos Spiritus sanctus posuit regere ecclesiam Dei, presbyteris tam ordine quam iurisdictione ex divina institutione superiores esse. Neque enim presbyteris, sed episcopis tantum competit, sacerdotes aliosque ministros ordinare, atque ecclesias sibi commissas propria et ordinaria protestate regere. Itaque et singulti in sua quisque eccelsia et congregati in synodis de doctrina et disciplina decernunt, leges ferunt, iudicium exercent. Neque fas est presbyteris sive aliis clericis suo in gradu et munere quidquam sine antistitis auctoritate agere: ut ecclesia super episcopos constituatur, et omnis actus ecclesiae per eosdem praepositos gubernetur (S. Cypr.). Verum etiam supremi muneris docenti et gubernandi universam ecclesiam episcopi expertes non sunt. Illud enim ligandi et solvendi pontificium, quod Petro soli datum est, collegio quoque apostolorum, suo tamen capiti coniuncto, tributum esse constat, protestante Domino: 'Amen dico vobis, quaecumque aligaveritis super terram, erunt ligata et in caelo; et quaecumque solveritis super terram, erunt soluta et in caelo' (Matth.XVIII, 18). Quapropter inde ab ecclesiae primordiis oecumenicorum conciliorum decreta et statuta iure merito tanquam Dei sententiae et Spiritus sancti placita summa veneratione et pari obsequio a fidelibus suscepta sunt. At quondam primatus Petro datus est, ut una ecclesia Christi et cathedra una monstraretur, Romano pontifici ceteri praesules subiecti sunt, tum singuli in propriis ecclesiis administrandis, tum universi in communibus ecclesiae negotiis gerendis. Ad summum enim hierarcham pertinet, novas ecclesias instituere, iam institutas aliis finibus circumscribere aut prorsus abolire, singulis proprios patores vel eligere vel electos confirmare, horum potestatem ordinariam ampliare et restringere, acta sive singulorum sive synodorum diiudicare, ipsos quoque praesules, ubi opus est, a munere removere. Neque hi pro universali ecclesia quidquam disponete vel secernere possunt, nisi ab regnante pontifice in partem 
der Bischöfe zu bestreiten. Im Gegenteil: Die Konzilsväter wollten eigentlich zeigen, dass die Vollmacht des römischen Bischofs die ordentliche und unmittelbare Vollmacht aller anderen Bischöfe nicht aufhebt, sondern sie vielmehr schützt, bejaht und stärkt. Die Konzilsväter haben ja schliesslich die Bischöfe als Nachfolger der Apostel und als wahre Hirten bezeichnet ${ }^{41}$, so wie es eigentlich ausdrücklich die Konstitution Pastor aeternus voraussieht ${ }^{42}$.

Die Frage, die auf dem Konzil im Bezug auf Papst und Bischöfe entstand, war: wie können eigentlich zwei bischöfliche Jurisdiktionen über dieselben Gläubigen praktiziert werden, ohne dass sie miteinander konkurrieren? Diese Frage bestimmte allerdings die Debatten im Konzil, die letztendlich allein um die potestas jurisdictionis und nicht um die potestas ordinis kreisten. Wie vorher gezeigt, stand bereits auf dem Konzil von Trient das umstrittene und leider nicht genug geklärte Problem des Ursprungs der bischöflichen Jurisdiktion zur Debatte. Die Frage die aus dieser Problematik damals und auch auf dem I. Vatikanum entstand, war, ob in der Relation der Unterordnung aller Bischöfen unter dem Papst auch eine Ursprungsbeziehung implizit gegeben ist. Obwohl die Tendenz der Konzilsväter auf dem I. Vatikanum war, den römischen Bischof als unmittelbare Quelle jeder jurisdictio episcopalis zu verstehen, lehnte das Konzil eine Entscheidung darüber ab. So wie dieses Problem sich den Vätern in Trient in der Frage der Begründung der

sollicitudinis vocati: et licet, ab eo congregati, tanquam veri iudices et fidei decreta et disciplinae leges condant; Romani tamen pontificis est, generalia eorum concilia non solum convocare et dissolvere, sed etiam dirigere et confirmare" (Hervorhebungen von mir).

${ }^{41}$ Vgl. G. BAUSENHART, Theologischer Kommentar zum Dekret über die Hirtenaufgabe der Bischöfe in der Kirche "Christus Dominus " 233.

${ }^{42}$ Die dogmatische Konstitution „Pastor aeternus “ über die Kirche Christi, in: DH 3061: „So wenig aber beeinträchtigt diese Vollmacht des Papstes jene ordentliche und unmittelbare Vollmacht der bischöflichen Jurisdiktion, mit der die Bischöfe, die, eingesetzt vom Heiligen Geist (vgl. Apg 20,28), an die Stelle der Apostel nachgefolgt sind als wahre Hirten die ihnen jeweils zugewiesenen Herden jeweils weiden und bitten, dass sie vielmehr vom obersten und allgemeinen Hirten bejaht, gestärkt und geschützt wird (...)“ (Hervorhebungen von mir). 
Residenzpflicht der Bischöfe stellte, und zwar im Zusammenhang mit dem Verdacht eines antipäpstlichen Konziliarismus - der Papst verbot ja am 08.05.1562 jegliche Diskussion über die Residenzpflicht der Bischöfe ${ }^{43}$-, war dies nicht mehr der Fall auf dem I. Vatikanum. Die Beschlüsse des I. Vatikanischen Konzils waren aber sehr wichtig, nicht nur aus rein ekklesiologischer Sicht, sondern auch bezüglich der sogenannten politischen Dimension des Bischofsamtes, also bezüglich der Unabhängigkeit des Bischofsamtes gegenüber den Instanzen weltlicher Macht. Worum ging es eigentlich? Die Konzilsbeschlüsse haben im damaligen deutschen Reich einen stürmischen Protest ausgelöst, der sich durch ein Schreiben vom Reichskanzler Otto von Bismarck konkretisiert hat. Auf dieses Schreiben haben die deutschen Bischöfe reagiert mit einer konkreten Antwort, in der man an der Unabhängigkeit des Bischofsamtes festhält. Die Bischöfe sind kraft göttlicher Einsetzung unabhängig gegenüber dem Staat im politischen Sinne und zugleich auch keine Beamte des Papstes. Durch die Beschlüsse des I. Vatikanischen Konzils ist die bischöfliche Jurisdiktion nicht in der päpstlichen aufgegangen, sondern sie wurde gestärkt und beschützt. Noch mehr: der Papst hat weder das Recht, noch die Macht diese von Gott selbst getroffene Anordnung zu ändern ${ }^{44}$. Eigentlich hatte das Konzil gallikanische Positionen im Blick als es diese Beschlüsse

43 Vgl. G. BAUSENHART, Theologischer Kommentar zum Dekret über die Hirtenaufgabe der Bischöfe in der Kirche "Christus Dominus " (S. dazu auch: Anm. 39) 234.

${ }^{44}$ Antworten auf die Circular-Depesche des Reichskanzlers Bismark über die Auslegungbder onstitution „Pastor aeternus“ des 1. Vatikanischen Konzils, Jan.-März 1875: a) Gemeinsame Erklärung de Bischöfe Deutschlands, Jan.Febr. 1875, in: DH 3112-3117 (hier 315): „Kraft derselben göttlichen Einsetzung, worauf das Papsttum beruht, besteht auch der Episkopat. Auch er hat seine Rechte und Pflichten vermöge der von Gott selbst getroffenen Anordnung, welche zu ändern der Papst weder das Recht, noch die Macht hat. Es ist also ein völliges Missverständnis der Vatikanischen Beschlüsse, wenn man glaubt, durch dieselben sei 'die bischöfliche Jurisdiktion in der päpstlichen aufgegangen', der Papst sei 'im Princip an die Stelle jedes einzelnen Bischofs getreten', die Bischöfe seien nur noch 'Werkzeuge des Papstes, seine Beamten ohne eigene Verantwortlichkeit'(...).“ 
verfasst hat, und deswegen galt die Sorge der Majorität auf dem Konzil dem Päpstlichen Primat ${ }^{45}$.

Die Problematik des Verhältnisses der beiden Jurisdiktionen tauchte aber auch später wieder auf, besonders im Vorfeld des II. Vatikanischen Konzils. So z.B. machte sich Papst Pius XII. in seiner Enzyklika Mystici Corporis ${ }^{46}$ diese Problematik zu Eigen ${ }^{47}$, indem er die Lehre der päpstlichen Jurisdiktion als Quelle jeder bischöflichen erneut zur Geltung bringt. Laut seiner Konzeption werden die Teilkirchen, genau wie die gesamte Kirche

„von Christus Jesus durch die Stimme und Vollmacht des eigenen jeweiligen Bischofs geleitet. Deshalb sollen die Bischöfe nicht nur für herausragendere Glieder der gesamten Kirche gehalten werden, weil sie durch ein ganz einzigartiges Band mit dem göttlichen Haupt des ganzen Leibes verbunden sind und daher zurecht 'erste Teile der Glieder des Herrn ${ }^{48}$ genannt werden, sondern - was die eigene Diözese eines jeden anbelangt - weil sie als wahre Hirten im Namen Christi die ihnen jeweils zugewiesenen Herden jeweils weiden und leiten ${ }^{49}$; indem sie jedoch dies tun sind sie nicht völlig eigenen Rechts (id tamen dum faciunt, non plane sui iuris sunt), sondern unterstehen der gebührenden Autorität des Römischen Bischofs (sed sub debita Romani Pontificis auctoritate positi), obwohl sie die ordentliche Jurisdiktionsvollmacht genießen, die ihnen von ebendiesem Papst unmittelbar zugeteilt wurde (quamvis ordinaria iurisdictionis potestate fruantur, immediate sibi ab eodem Pontifice Summo

45 Vgl. G. BAUSENHART, Theologischer Kommentar zum Dekret über die Hirtenaufgabe der Bischöfe in der Kirche "Christus Dominus " 233.

${ }^{46}$ Lateinischer integraler Text in: AAS 35 (1943) 211-212. Integraler deutscher Text in: Rundschreiben unseres Heiligen Vaters PAPST PIUS XII. „,Mystici Corporis Christi"/Über den mystischen Leib Jesu Christi und unsere Verbindung mit Christus in ihm (Luzern $\left.{ }^{8} 1960\right)$ 5-46.

47 Vgl. G. BAUSENHART, Theologischer Kommentar zum Dekret über die Hirtenaufgabe der Bischöfe in der Kirche "Christus Dominus" 235.

${ }^{48}$ PAPST PIUS XII. begründet diese Aussage mit Hinweis auf PAPST GREGOR I., Moralia XIV, 35,43, in: PL 75, 1062B/M. ADRIAEN: CpChL 143A [1979] $724_{36}$.

49 Der Text der Enzyklika weist an dieser Stelle auf das Erste Vatikanische Konzil, und zwar auf die Dogmatische Konstitution „Pastor aeternus “ über die Kirche Christi. S. dazu: DH 3061. 
impertita). Deshalb sind sie als Nachfolger der Apostel aufgrund göttlicher Einsetzung (ex divina institutione) vom Volk zu verehren" ${ }^{, 50}$.

Auf jeden Fall zeigt die Frage der beiden Jurisdiktionen deutlich, dass das Verhältnis zwischen dem Papst und den einzelnen Bischöfen sehr stark im Bewusstsein der Konzilsväter bleibt, während die Konzeption über das Bischofskollegium eine marginale Sache ist. Die Situation ändert sich aber radikal auf dem II. Vatikanischen Konzil, denn auch wenn die bischöfliche Perspektive immer noch auf den Papst und seinen Primat gerichtet ist, will das Konzil die Einheit zwischen dem Papst und dem Bischofskollegium viel deutlicher ins Licht stellen ${ }^{51}$. Insofern kann die Kirchenkonstitution Lumen Gentium behaupten, dass das Konzil nichts anderes sein will und kann als die Fortsetzung des I. Vatikanums. Aber als dessen Fortsetzung ergänzt das Konzil, was auf dem I. Vatikanum fehlt, und zwar verbindet es inhaltlich die Lehre über das Bischofsamt mit der Lehre über den Papst ${ }^{52}$. In diesem Sinne proklamiert das Konzil Folgendes:

Um Gottes Volk zu weiden und immerfort zu mehren, hat Christus der Herr in seiner Kirche verschiedene Dienstämter eingesetzt, die auf das Wohl des ganzen Leibes ausgerichtet sind. Denn die Amtsträger, die mit heiliger Vollmacht ausgestattet sind, stehen im Dienste ihrer Brüder, damit alle, die zum Volke Gottes gehören und sich daher der wahren Würde eines Christen erfreuen, in freier und geordneter Weise sich auf das nämliche Ziel hin ausstrecken und so zum Heile gelangen. Diese Heilige Synode setzt den Weg des ersten Vatikanischen Konzils fort und lehrt und erklärt feierlich mit ihm, daß der ewige Hirt Jesus Christus die heilige Kirche gebaut hat, indem er die Apostel sandte wie er selbst gesandt war vom Vater (vgl. Joh 20, 21). Er wollte, daß deren

${ }^{50}$ PIUS XII., Enzyklika „Mystici corporis“, in: DH 3804. Vgl. dazu auch: Rundschreiben unseres Heiligen Vaters PAPST PIUS XII. „,Mystici Corporis Christi "/̈ber den mystischen Leib Jesu Christi und unsere Verbindung mit Christus in ihm (§ 41) 18f.

${ }^{51}$ Vgl. dazu auch: G. BAUSENHART, Theologischer Kommentar zum Dekret über die Hirtenaufgabe der Bischöfe in der Kirche "Christus Dominus " $235 \mathrm{f}$.

${ }^{52}$ Vgl. W. BreunING, Das Verständnis des katholischen Bischofsamtes $10 \mathrm{f}$. 
Nachfolger, das heißt die Bischöfe, in seiner Kirche bis zur Vollendung der Weltzeit Hirten sein sollten. Damit aber der Episkopat selbst einer und ungeteilt sei, hat er den heiligen Petrus an die Spitze der übrigen Apostel gestellt und in ihm ein immerwährendes und sichtbares Prinzip und Fundament der Glaubenseinheit und der Gemeinschaft eingesetzt. Diese Lehre über Einrichtung, Dauer, Gewalt und Sinn des dem Bischof von Rom zukommenden heiligen Primates sowie über dessen unfehlbares Lehramt legt die Heilige Synode abermals allen Gläubigen fest zu glauben vor. Das damals Begonnene fortführend, hat sie sich entschlossen, nun die Lehre von den Bischöfen, den Nachfolgern der Apostel, die mit dem Nachfolger Petri, dem Stellvertreter Christi und sichtbaren Haupt der ganzen Kirche, zusammen das Haus des lebendigen Gottes leiten, vor allen zu bekennen und zu erklären (LG 18).

Die Frage nach der Stellung des Bischofsamtes in der Kirche wurde vor allem unter dem Aspekt der Communio, der Gemeinschaft der Bischöfe untereinander und mit dem Papst behandelt, und infolgedessen wurde die Lehre über das Bischofskollegium ins Zentrum der Theologie des Konzils über das Dienstamt gestellt. Für katholisches Kirchenverständnis ist das Bischofsamt nicht einfach eine äusserliche organisatorische Grösse, sondern es ist die innere Kraft, die dort, wo Kirche Communio ist, immer neu Kirche aufbaut. Deshalb hat das II. Vatikanische Konzil auch festgestellt, dass die Verfassung der Kirche nicht in einem Antagonismus zwischen den Bischöfen und dem Papst begriffen werden darf. Die mittelalterliche Kontroverse zwischen Papalismus und Konziliarismus hatte in den Augen der Konzilsväter keinen Gegenstand mehr. Das Konzil suchte einen anderen Weg aus dieser Problemstellung heraus, indem es die Frage nach der Machtausübung innerhalb einer Kirchenverfassung der Communio stellte ${ }^{53}$. Aus dieser Hinsicht kann man behaupten, dass das II. Vatikanische Konzil für die ganze Christenheit und nicht nur für die katholische Kirche ein Ereignis von grosser Bedeutung war. Sein grosses Verdienst ist nicht nur, dass es den Inhalt des Selbstbewusstseins und die Identität der katholischen Kirche neu entdeckt und formuliert hat, sondern auch, dass es eine Brücke zur

${ }^{53}$ Vgl. W. BREUnING, Das Verständnis des katholischen Bischofsamtes 11-13. 
orthodoxen Kirche aufgebaut hat. Die neuen Tendenzen innerhalb der Orthodoxie - wie z.B. die Bestrebungen zur Einberufung eines panorthodoxen Konzils oder verschiedener panorthodoxer Konferenzen - zeigen, dass die nachkonziliaren Annährungen zwischen den beiden Teilen der Christenheit sehr bedeutsam sind und zugleich, dass sie einen effektiven Dialog bewirken ${ }^{54}$.

Dieser Dialog wird aber nur fruchtbar und immer fruchtbarer, wenn man das Wesentliche nicht aus den Augen verliert. Konkret: was das Bischofsamt anbelangt, muss man bereits von Anfang an feststellen, dass das Erreichen einer gemeinsamen Theologie die Notwendigkeit einer theologischen Zusammenschau voraussetzt. Der Versuch, eine völlig neue, moderne Theologie des Bischofsamtes herzustellen, die keinen Bezug oder keine Rücksicht auf die gemeinsame Tradition nimmt, wird möglicherweise zu einem für die Kirche unakzeptablen Verständnis des Bischofsamtes führen. Als wesentliches Element der Kirche, ohne die sie nicht existieren kann, gründet das Bischofsamt im göttlichen Willen, dessen Konkretisierung in der heilwirkenden Menschwerdung Jesu Christi zum Ausdruck kommt: Und das Wort ist Fleisch geworden und hat unter uns gewohnt, und wir haben seine Herrlichkeit gesehen, die Herrlichkeit des einzigen Sohnes vom Vater, voll Gnade und Wahrheit (Joh 1,14). Nur aufgrund dieses christologischen Geschehens, das zugleich auch pneumatologisch zu betrachten ist, kann man wieder $\mathrm{zu}$ einer gemeinsamen Theologie des Bischofsamtes kommen. Das Ereignis der Fleischwerdung des Wortes Gottes stellt unseren Augen und unserem Verstand das völlig Neue, das Unerhörte schlechthin vor. Das Wort wird von sich aus, von Gott her, bei dem es vor allen Zeiten existiert, Fleisch, und so enthüllt es uns in diesem Axiom das Wesen der endgültigen Offenbarung Gottes. Gott wird Fleisch, wird der konkrete Mensch,

${ }^{54}$ Vgl. D. PAPANDREOU, Vorwort, in: M. BRUN, Orthodoxe Stimmen zum II. Vatikanum. Ein Beitrag zur Überwindung der Trennung (= ÖBFZPhTh 18) (Freiburg i. Ü. 1988) III. 


\section{$15^{\text {th }}$ International Symposium on Science, Theology and Arts}

dessen Gegenwart und Erscheinungsweise das individuelle Leibgebilde ist ${ }^{55}$.

Die Kirche ist deswegen in ihrem Theologie-Treiben immer bewusst auf dem im christologisch-pneumatologischen Ereignis gekennzeichneten Weg gegangen. Die Theologie des Bischofsamtes kann und darf deshalb nicht ausserhalb des organisch-ontologischen Rahmens eines derartigen Grundsatzes verstanden werden. Der Übergang von Jesus Christus zum - in der Welt von ihm selbst und durch die von ihm eingesetzten Diener inkarnierten - Heil wurde und wird immer wieder in der Konkretisierung des Fleisches, d.h. in der Person, vollzogen. Jeder Bischof steht dadurch in einer Nachfolgekette, welche ihn in einer endgültigen und geheimnisvollen Weise aufgrund des in der Weihe, d.h. im sacramentum ordinis enthaltenen christologisch-pneumatologischen Geschehens kennzeichnet und determiniert. Schon das Konzil von Trient hat diese in der kirchlichen Tradition immer lebendige Sichtweise bestätig. Deswegen konnte man sich einigen und festhalten, dass nicht das Volk oder irgendeine weltliche Autorität durch Wahl oder Ablehnung, durch Ein- oder Absetzung jemanden zum Bischof und dann zurück zum Laien machen kann, sondern dass dies erst und nur durch die Weihe geschieht. Was aber in der Weihe geschieht, kann nicht mehr rückgängig gemacht werden, weil sie dem Empfänger einen unauslöschlichen sakramentalen Charakter (character indelebilis) verleiht, der eng an seine Person gebunden ist. Das zeigt, dass das sacramentum ordinis und damit auch das Bischofsamt nicht in sich betrachtet werden kann, sondern dass es immer zusammen mit seiner ekklesiologischen bzw. christologisch-pneumatologischen Verwurzelung verstanden werden muss. Der ekklesiale Auftrag des Aufbaues des Leibes Christi ist deswegen als eine an die Person des Bischofs gebundene Funktion zu verstehen. Deswegen werden die Gestalt des Bischofs und sein von seiner Person nicht getrenntes Wirken immer zum Ausgangs- und Mittelpunkt des richtigen Verständnisses seines Amtes. Der ordo ist ein von Christus eingesetztes und in sich gestuftes Sakrament. Er bildet ein Ganzes,

${ }^{55}$ H. U. vON BALTHASAR, Der antirömische Affekt 12. 
ist ein Dienst und kann nur von einem Bischof gespendet werden, denn nur der Bischof besitzt die Fülle der Teilhabe am Priestertum Jesu Christi. Deshalb und aufgrund des auf Gott selbst zurückgehenden Weiherechts des Bischofs ist die von ihm gespendete, in Gott verankerte und von Christus eingesetzte ordinatio sacerdotalis wirksam ${ }^{56}$.

Das Konzil von Trient hat gegen die damals neu entstandene protestantische Lehre ganz genau auf einen sehr wichtigen Aspekt, nämlich auf die Personalisierung des ordo gezielt, in dem Sinne, dass sich die in der Weihe dem Bischof erteilte potestas streng an seiner Person bemisst ${ }^{57}$.

Auch das I. bzw. das II. Vatikanische Konzil haben sich die in Trient behandelten Aspekte zu Eigen gemacht, wenngleich aufgrund der unterschiedlichen historischen Umstände nicht in gleicher Weise. Dennoch kann man ohne Zweifel feststellen, dass die getroffenen Entscheidungen nichts Widersprüchliches in sich haben. Im Gegenteil: Sie weisen auf eine volle Einheit der lehramtlichen Aussagen aller drei Konzilien und auf eine unbestreitbare Kontinuität mit der alten Kirche hin. Die Notwendigkeit einer theologischen Zusammenschau braucht deswegen keine weitere Begründung.

\section{Ergebnisse und Fragen}

Am Ende dieser kurzen Untersuchung über das Bischofsamt in der katholischen Kirche ergeben sich einige Schlussfolgerungen, Bemerkungen und Fragen, die sich in bezug auf die analysierten Aspekten folgendermassen formulieren lassen:

i) Warum war das Thema Bischofsamt für das II. Vatikanische Konzil wichtig?

ii) Was wollten die Konzisväter durch die zu diesem Thema entstandenen theologischen Reflexionen aussagen?

Als gelebte Realität der Katholischen Kirche hat das Bischofsamt zweifelsohne eine konstitutive Bedeutung für sie, denn

\footnotetext{
${ }^{56} \mathrm{Vgl}$. J. FreITAG, Sacramentum ordinis $115 \mathrm{f}$.

${ }^{57}$ Vgl. ebd. 124f.
} 
ohne dieses im kirchenstiftenden Leben und Willen des Herrn begründete Amt kann die Kirche nicht Kirche sein. Man muss aber von Anfang an deutlich feststellen, dass eine solche Begründung nicht als ein exklusiv juristischer Akt interpretiert werden darf, sondern sie ist vielmehr inhaltlich auf Christus zurückzuführen. Deswegen muss jede Beschreibung dieses Amtes in das Leben der Kirche und in ihre Geschichte hineinschauen, denn die Kirche selbst hat beim Definieren der Lehre über das Bischofsamt das kirchliche Leben und seine Entfaltung als Grundlage genommen. Dabei hilft aber vor allem das, was vom II. Vatikanischen Konzil über das Bischofsamt in theologischer Reflexion lehrmässig gesagt wurde ${ }^{58}$. Das von den Konzilsvätern behandelte Thema Bischofsamt ruft in Erinnerung, dass das II. Vatikanische Konzil keine neue Lehre über die Kirche und die dazu gehörende hierarchische Struktur entwickelt hat, sondern aufgrund der während des Konzils innerhalb der verschiedenen Schemata entstandenen theologischen Reflexionen die aus der Tradition ergebene und gelebte dogmatische Lehre über das Bischofsamt entfalten und vertiefen wollte. Die in zwei wichtigen Konzilsdokumenten - Die dogmatische Konstitution über die Kirche 'Lumen Gentium' und Das Dekret über die Hirtenaufgabe der Bischöfe 'Christus Dominus' - enthaltenen Aussagen der Konzilsväter bezeugen, dass eine richtige Theologie des Bischofsamtes nur vom Wesen der Kirche her richtig verstanden wird, und dass die konkrete Existenzform der katholischen Kirche ihren wahren Ausdruck im Bischofsamt findet. Dort wo der Bischof ist, ist Ecclesia, und dort wo die Ecclesia ist, ist auch das Bischofsamt gegeben. Auf diese Wiese zielt die Theologie des Bischosamtes auf die Personalisierung des ordo in dem Sinne, dass sich die in der Weihe dem Bischof erteilte potestas streng an seiner Person bemisst. Noch mehr: Im Bischofsamt verwirklicht sich die Vollständigkeit der sakramentalen Struktur der Kirche, so dass das Bischofsamt sich als konstitutives Element des katholischen Kirchenkonzeptes erweist. Aufgrund dieser Überlegungen und im

${ }^{58}$ Vgl. W. BREUnING, Das Verständnis des katholischen Bischofsamtes 9. 
Zusammenhang mit den Ergebnissen des Trienter- bzw. des I. Vatikanischen Konzils, ergeben sich drei weitere Fragen:

iii) Bilden die Beschlüsse dieser Konzilien über das Bischofsamt eine Einheit oder widersprechen sich?

iv) Wie verhalten sich Entwicklung, Kontinuität und Ergänzungsnotwendigkeit zueinander?

v) Können diese drei Konzepte zu einem notwendigen theologischen Zusammenschau führen?

Um zu einer Schlussfolgerung der Beschlüssen der drei Konzilien über das Bischofsamt kommen zu können, stellt man zunächst fest, dass ihre wichtigsten Aussagen im Grunde genommen übereinstimmen. Natürlich hat jedes Konzil seine Entscheidungen auch aufgrund der konkreten historischen Lage getroffen und sie dieser Situation entsprechend formuliert. Man kann aber ohne Zweifel behaupten, dass die Basis der heutigen dogmatischen Aussagen der katholischen Kirche über das Bischofsamt tief im Konzil von Trient wurzelt, das sich seinerseits nur als Kontinuität zur alten Kirche und zu ihrer Tradition verstand. Dass das Bischofsamt auf dem II. Vatikanischen Konzil als Dienst angesehen wurde, war eigentlich nichts Neues, denn auch das Konzil von Trient hat das in einem bestimmten Rahmen festgelegt. Als auf dem II. Vatikanischen Konzil die Aussagen über das Verhältnis zwischen Papst und Bischöfen erneut zur Debatte kamen, geschah damit nichts Neues, denn auch das I. Vatikanum hat sich darum gekümmert, wenngleich aufgrund der damaligen historischen Lage nicht vollständig. Vielmehr kann man behaupten, dass jedes Konzil durch seine Aussagen die vorgehenden bestätigt hat, denn alle verstehen sich in einer und derselben Kontinuität zur alten Kirche und zu ihrer Tradition. Ergänzungsnotwendigkeit schliesst diese Kontinuität nicht aus, denn die Kirche findet auf ihrem Weg immer neue Ausdrucksmöglichkeiten der einen und selben Wahrheit, weil Kirche eben ständig der Erneuerung bedarf - ecclesia semper reformandaund weil ihre Tradition nicht etwas Statisches sondern etwas Lebendiges ist, das eng an die Person ihres Gründers, Jesus Christus, der immer in seiner Kirche lebt, gebunden ist. 


\section{Literaturverzeichnis}

1. Die dogmatische Konstitution „Pastor aeternus “ ̈̈ber die Kirche Christi, in: DH 3050-3075.

Schema constitutionis dogmaticae secundae de ecclesia Christi secundum reverendissimorum animadversiones reformatum (Constitutio secunda), in: MANSI 53, 308A317A.

2. Die Antworten auf die Circular-Depesche des Reichskanzlers Bismarck über die Auslegung der Konstitution "Pastor aeternus" des I. Vatikanischen Konzils, Januar - März 1875:

a) Gemeinsame Erlärung der Bischöfe Deutschlands, Jan. - Febr. 1875 und

b) Apstolisches Schreiben „Mirabilis illa constantia“ an die Bischöfe Deutschlands, 4. März 1875, in: DH 31123117 . 